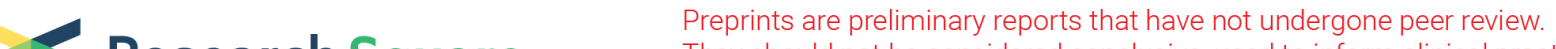 Research Square They should not be considered conclusive, used to inform clinical practice, or referenced by the media as validated information.
}

\section{Potential Biomarkers for Predicting the Overall Survival of Lung squamous cell carcinoma: A analysis of Ferroptosis-Related IncRNAs}

\section{zixuan Wu}

Guangzhou University of Traditional Chinese Medicine: Guangzhou University of Chinese Medicine

\section{Xuyan Huang}

Guangzhou University of Traditional Chinese Medicine: Guangzhou University of Chinese Medicine

\section{Min-jie Cai}

Guangzhou University of Traditional Chinese Medicine: Guangzhou University of Chinese Medicine

Peidong Huang ( $\sim$ yeruyun@163.com )

Yunnan University of Traditional Chinese Medicine https://orcid.org/0000-0002-3044-9269

\section{Zunhui Guan}

Kunming Municipal Hospital of Traditional Chinese Medicine

\section{Research}

Keywords: Lung squamous cell carcinoma (LUSC), ferroptosis-related IncRNAs, TCGA datasets, immune cell infiltration, Bioinformatics analysis

Posted Date: October 18th, 2021

DOI: https://doi.org/10.21203/rs.3.rs-958875/v1

License: (c) (i) This work is licensed under a Creative Commons Attribution 4.0 International License. Read Full License 


\section{Abstract \\ Background}

In 502 Lung squamous cell carcinoma (LUSC) samples from The Cancer Genome Atlas (TCGA) datasets, the predictive significance of ferroptosis-related long non-coding RNAs (IncRNAs) was investigated. In LUSC, we meant to express how ferroptosis-associated IncRNAs interact with immune cell infiltration.

\section{Methods}

Gene expression enrichment was investigated using gene set enrichment analysis in the Kyoto Encyclopedia of Genes and Genomes. The prognostic model was constructed using Lasso regression. To better understand immune cell infiltration in different risk groups and its relationship to clinical outcome, researchers analyzed by modifications in the tumor microenvironment (TME) and immunological association. The expression of IncRNA was intimately connected to that of ferroptosis, according to coexpression analyses. Ferroptosis-related IncRNAs were shown to be partially overexpressed in high-risk patients in the absence of additional clinical signs, suggesting that they may be incorporated into a prediction model to predict LUSC prognosis. GSEA revealed the immunological and tumor-related pathways in the low-risk group.

\section{Results}

According to TCGA, CCR and inflammation-promoting genes were considered to be significantly different between the low-risk and high-risk groups. The expression of C10orf55, AC016924.1, AL161431.1, LUCAT1, AC104248.1, and MIR3945HG were likewise different in the two risk groups.

\section{Conclusion}

LncRNAs linked to ferroptosis are connected to the occurrence and development of LUSC. With the use of matching prognostic models, the prognosis of LUSC patients can be predicted. In LUSC, ferroptosisrelated IncRNAs and immune cell infiltration in the TME might be novel therapeutic targets that should be investigated further.

\section{Background}

Lung cancer is among the most frequent cancers in the world, killing about 500,000 people in China each year ${ }^{\square}$. The most frequent kind of lung cancer is lung squamous cell carcinoma (LUSC), which accounts for $40-51 \%$ of all primary lung malignancy ${ }^{\square}$. The prevalence of LUSC is strongly related to age, gender, and smoking. LUSC developed slowly, metastasized late, was more likely to be surgically respected in the 
middle and early stages, and had a higher 5 -year survival rate ${ }^{\rrbracket}$. Small cell undifferentiated carcinoma was more susceptible to radiotherapy and chemotherapy than LUSC. Recent developments in molecular research and the development of new medications targeting molecular anomalies have largely driven advances in the treatment of LUSC. Existing therapy targets, on the other hand, are susceptible to resistance ${ }^{\square}$. Therefore, novel predictive biomarkers for the diagnosis, prognosis, and therapy of LUSC are so urgently needed.

LncRNAs are RNA molecules that have a high degree of expression selectivity. Several studies have discovered that IncRNAs have a variety of biological roles, including gene control, cancer, development, and even metastasis regulation ${ }^{[-]}$. Iron droop in tumor cells has received a lot of interest in recent years as a novel cell death that can help tumor cells escape therapeutic resistance ${ }^{[-]}$. In contrast to apoptosis and autophagy, iron failure, which is an iron dependent and reactive oxygen species (ROS)-dependent cell death, can be used to treat a variety of disorders. Cancer cells are more iron dependent than normal cells and rely on iron excessively to proliferate. Hence an imbalance in iron metabolism may hasten tumor growth $^{\square}$. There is no question that activating the Ferroptosis pathway could overcome resistance to present chemotherapeutic drugs and expand the boundaries of cancer therapy ${ }^{\square}$. The relationship between IncRNA and immune cell infiltration in ironophilic cell illness, on the other hand, is still unknown.

Depending on research, IncRNA can regulate iron droop and control iron death and apoptosis, whereas silencing IncRNA can greatly reduce iron droop and regulate inflammation and lipid peroxidation ${ }^{[-]}$. Nonetheless, sequencing investigations of aberrant IncRNA expression and its relationship to overall survival (OS) in iron-addicted LUSC patients are uncommon.

Immune checkpoint-associated gene profiles in LUSC patients may be beneficial in detecting therapy response, assessing risk, and predicting survival ${ }^{\square}$. Despite the fact that little study has been conducted on the association between iron-cytopathic-associated Incrnas and immune cell infiltration in LUSC, it is critical to explore immune cell infiltration in TME and its relationship with clinicopathological characteristics of LUSC tumors. There is limited research on the causes and mechanisms of aberrant IncRNA expression and iron droop in LUSC at the moment. To further understand the IncRNA-related pathways that alter the prognosis of LUSC patients, a transcription map of IncRNA expression and ferroptosis changes in LUSC patients is required. Furthermore, immune checkpoint-associated gene profiles can be used as predictors of therapeutic response to LUSC patients to assess risk and predict overall survival.

The purpose of this work was to identify ferroptosis-related IncRNAs whose expression is linked to LUSC patients' prognosis in order to develop a predictive model for LUSC prognosis prediction. To aid in the identification of novel LUSC therapeutic targets and pharmacological options by better understanding the infiltration of ferroptosis-related IncRNAs and their associated immune cells in TME.

Figure 1. Framework based on an integration strategy of ferroptosis-related IncRNAs. 


\section{Marerials And Methods}

\subsection{Datasets and ferroptosis-related Genes}

Using Genomic Data Commons Data Portal (https://portal.gdc.cancer.gov/) " , we collected LUSC gene expression patterns and clinical data from the Cancer Genome Atlas (TCGA). The strategy is to investigate at gene expression profiles (Cases: bronchus and lung, TCGA and TCGA-LUSC; Files: transcriptome profiling and Gene Expression Quantification and HTSeq-FPKM). Information on clinical research (Cases: bronchus and lung, TCGA and TCGA-LUSC; Files: clinical and bcr xml). The expression patterns of 502 cases of LUSC and 49 cases of normal tissues were enrolled in the TCGA shared database on October 5,2021 . Table 1 summarizes the clinical features of the patients. In addition, corresponding ferroptosis-related genes were downloaded from FerrDb $\llbracket$, a web-based consortium that provided a comprehensive and up-to-date database for ferroptosis markers, their regulatory molecules and associated diseases. We identified 382 ferroptosis-related genes (driver: 150; suppressor: 109; marker: 123) in total (Table S1).

Table 1

The clinical characteristics of patients in the TCGA dataset.

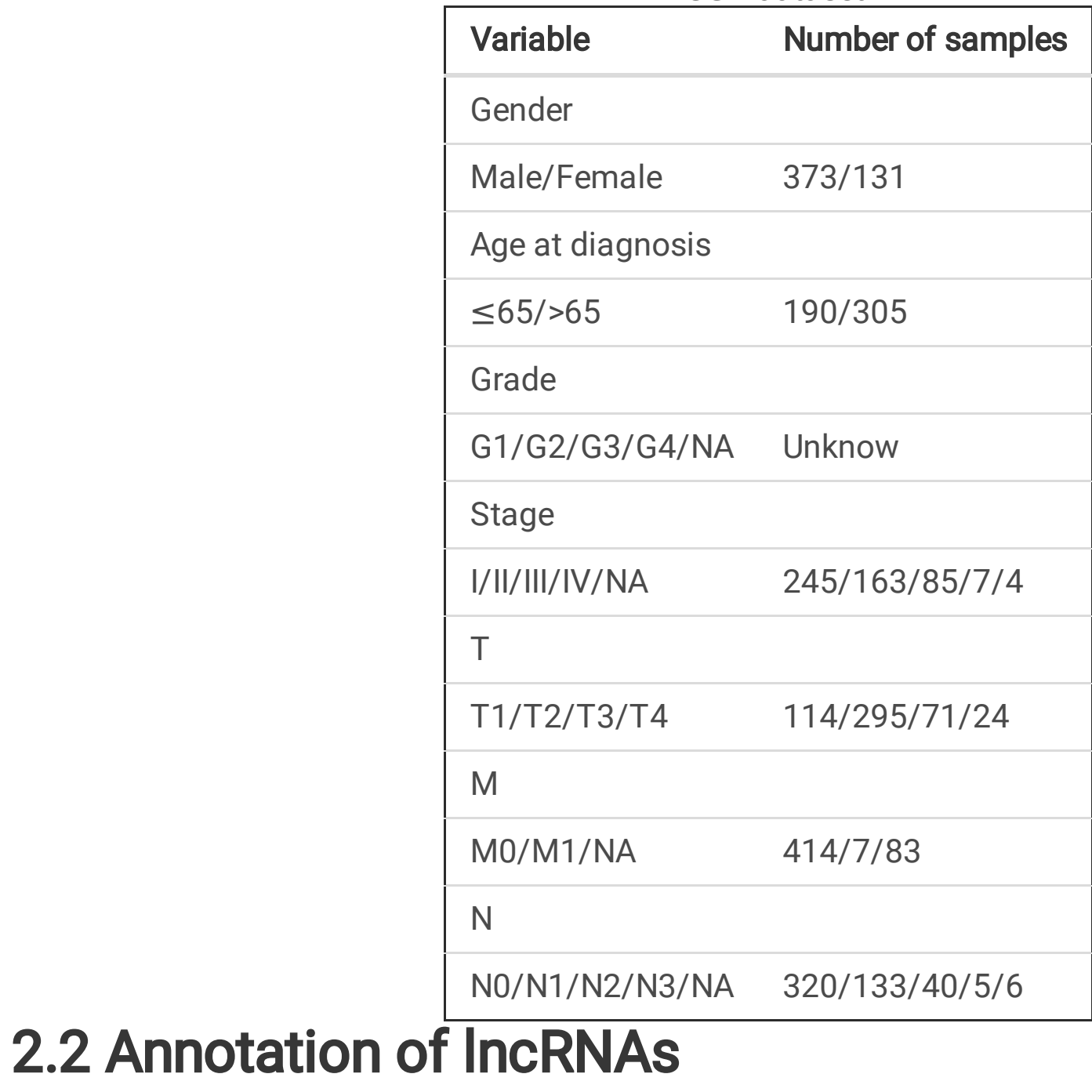


For annotation of the IncRNAs in the TCGA dataset, Genome Reference Consortium Human Build 38 (GRCh38) IncRNA annotation file was obtained from the GENCODE website ${ }^{4}$. With the help of perl software (https://www.perl.org/), the transcriptome data and human configuration files were matched and sorted, and the appropriate mRNA and IncRNA gene expression data were obtained. The gene IDs were translated to gene names using information from the ensemble database (http://asia.ensembl.org/info/data/index.html). The R4.1.0 Limma package was used to extract ferroptosis-related gene expression data, which was based on the gene expression matrix of ferroptosisrelated IncRNA gene expression profile data collected before.

\subsection{Identification of ferroptosis-related IncRNAs}

The relationship between ferroptosis-related IncRNAs and LUSC was investigated using Pearson correlation. After removing the normal samples and using $\mathrm{p}<0.001$ and corFilter $=0.4$ as screening criteria, the Limma package's correlation test was performed to evaluate the expression of ferroptosis-related IncRNA. Co-expression analysis was utilized to look at the relationship between ferroptosis-related gene expression and IncRNAs. The clinical-pathological information acquired from LUSC patients included gender, age, stage, grade, TMN, survival status, and survival time. To determine whether there was a significant difference in expression of ferroptosis-related IncRNAs, FDR $<0.05$ and $\mid \log 2 \mathrm{FCl} \geq 1$ were utilized. First, we investigated into the function of ferroptosis-related differentially expressed genes that were both upregulated and downregulated (DEGs). The biological pathways connected with the DEGs were then analysed using Gene Ontology (GO). Biological processes (BP), molecular functions (MF), and cellular components (CC) regulated by the differently expressed ferroptosis-related IncRNAs were further analyzed using R software, clusterProfiler, org.Hs.eg.db, enrichplot, and ggplot2 package based on Kyoto Encyclopedia of Genes and Genomes (KEGG) data.

\subsection{Development of the ferroptosis-related IncRNAs prognostic signature}

To build a prognostic model, Ferroptosis-related IncRNAs signature was constructed using Lassopenalized Cox regression and Univariate Cox regression analysis, stratified by risk score (Coefficient IncRNA $1 \times$ expression of IncRNA 1$)+($ Coefficient IncRNA $2 \times$ expression of IncRNA 2$)+\cdots+($ Coefficient IncRNA $A_{n} \times$ expression IncRNA $A_{n}$ ). Each LUSC patient's associated risk score was further evaluated. Based on the median score, the RNAs were divided into three subgroups: low-risk (< median number) and highrisk ( $\geq$ median number). In Lasso regression, the low-risk (50\%) and high-risk (50\%) groups were identified, and the corresponding plots were obtained. The confidence interval and risk ratio were calculated after visualization, and the forest diagram was constructed. The high-risk and low-risk groups' survival curves were constructed and compared. We utilized the timeROC software to design a comparable receiver-operating characteristics (ROC) curve to examine the accuracy of our model for predicting survival in LUSC. The risk and survival status of ferroptosis-related IncRNAs was investigated in connection to the risk curve, which was generated using the risk score. An independent prognosis analysis was performed to check whether our model was unaffected by other clinical prognostic factors 
that influence the patients' outcome. The researchers used multivariate and univariate models to calculate hazard ratios. To determine the association between clinical characteristics and our prediction risk model, as well as to distinguish between high-risk and low-risk ferroptosis-related cases. Risk and clinical correlation analyses were completed. Heatmap and limma packages were used to construct the Heatmap. To further demonstrate the correctness of our model, Decision Curve Analysis (DCA) was constructed.

\subsection{GSEA enrichment analyses and the predictive nomogram}

GSEA (https://www.gsea-msigdb.org/gsea/index.jsp) was used to discover variations in linked functions and pathways in diverse samples, and data was imported using the PERL programming language. Associated score and graphs were used to see if the functions and routes in various Risk groups were dynamic(c2.cp.kegg.v.7.2.symbols.gmt,Risk.cls\#h versus I). Depending on it was a high-risk cluster of prognosis-related IncRNAs, each sample was labeled as ' $\mathrm{H}$ ' or 'L'. The number of permutations, no collapse, and phenotype were set to 1000 , no collapse, and phenotypic, respectively. The gene list was sorted in 'real' mode, with the order of the genes in 'descending' mode. The 'Signal2Noise' measure was utilized to rank the genes. The normalization method was 'meandiv,' and the difference was statistically significant with a FDR<0.05. A nomogram was constructed integrating the prognostic signatures, for predictive of 1, 2 and 3 year OS of LUSC patients.

\subsection{Immunity analysis and gene expression}

Simultaneously, the CIBERSORT ${ }^{[-]}$, ESTIMATE ${ }^{\square}$, MCPcounter ${ }^{\square}$, single-sample gene set enrichment analysis (sSGSEA) ${ }^{\square}$, and TIMER ${ }^{\square}$ algorithms were compared to evaluate cellular components or cell immune responses between high and low risk groups based on ferroptosis-related IncRNA signatures. A Heatmap was utilized to discover changes in immune response under different algorithms. In addition, SSGSEA was utilized to compare and quantify the tumor-infiltrating immune cell subgroups in both groups, as well as their immunological function. Previous literature was designed to identify a possible immunological roadblock.

\subsection{Statistical analysis}

The data was analyzed using Bioconductor programs in $\mathrm{R}$ software version 4.1.0. To investigate normally and non-normally distributed variables, the Wilcoxon test and the unpaired student's t-test were utilized. The Benjamini-Hochberg technique was used to determine the variable expressed IncRNAs based on FDR. Utilizing "GSVA" and sSGSEA-normalized LUSC DEGs, the LUSC DEGs were compared to a genome (Rpackage). The sensitivity and specificity of the LUSC generate prognostic signals in comparison to other clinicopathological factors were evaluated using the operating characteristic curve (ROC) and decision curve analysis (DCA). The connection between ferroptosis-related IncRNAs and clinicopathological symptoms was investigated using logistic regression analysis and a heatmap graph. Based on the ferroptosis-related IncRNAs signature, the Kaplan-Meier survival analysis was used to estimate the survival of LUSC patients. For each analysis, statistical significance was identified as $\mathrm{P}<0.05$. 


\section{Results}

The purpose of this study was to illustrate how immune cell infiltration and ferroptosis-related IncRNAs influence LUSC. We identified 102 ferroptosis-related DEGs and 8 risk ferroptosis-related IncRNAs based on expression differences between tumor and normal tissues. GSEA was performed to discover latent signaling pathways that could be implicated in the development and progression of LUSC, and lasso regression was used to generate a suitable prognostic model.

\subsection{Enrichment Analysis of ferroptosis-related genes}

We discovered 102 DEGs linked to ferroptosis (35 downregulated and 67 upregulated; Table S2). 655 core targets were discovered by GO enrichment analysis, including MF, CC, and BP. The MF mainly involves antioxidant activity (GO:0016209), heme binding (GO:0020037), iron ion binding (G0:0005506), oxidoreductase activity, acting on $\mathrm{NAD}(\mathrm{P}) \mathrm{H}$ (GO:0016651). The CC mainly involves apical part of cell (G0:0045177), apical plasma membrane (GO:0016324), basal plasma membrane (G0:0009925), basal part of cell (G0:0045178). The BP mainly involves response to extracellular stimulus (G0:0009991), response to nutrient levels (G0:0031667), intrinsic apoptotic signaling pathway (GO:0097193), neuron death (GO:0070997). In addition, the main signaling pathways were identified by KEGG enrichment analysis, revealed the over-expressed genes were mainly involved in Lipid and atherosclerosis (hsa05417), HIF-1 signaling pathway (hsa04066), Chemical carcinogenesis-reactive oxygen species (hsa 05208), Ferroptosis (hsa 04216), MicroRNAs in cancer (hsa05206) (Figure 2 and Table S3).

Figure 2. GO and KEGG analyses for ferroptosis-related differentially expressed genes. (a) GO (b) KEGG.

\subsection{The ferroptosis-based IncRNAs prognostic signature}

505 ferroptosis-related IncRNAs were discovered (Table S4). 8 significant ferroptosis-related IncRNAs were discovered in the univariate COX study, and these were included in the multivariate COX analysis. Overall, 8 different IncRNAs (C10orf55, AC016924.1, AL161431.1, AP006545.2, LUCAT1, AC104248.1, AL122125.1, MIR3945HG) were discovered to be independent LUSC prognostic indicators (Figure 3) (Table S5). As a consequence, we calculated risk scores for the IncRNAs and constructed a prognostic signature.

Figure 3. Forest plot of significant IncRNAs.

\subsection{Survival results and multivariate examination}

Depending on Kaplan-Meier analyses, the expression of high-risk IncRNA signatures was associated with poorer survival $(\mathrm{P}<0.001$, Figure $4 a)$. Meanwhile, the signature IncRNAs' AUC was 0.658 , indicating that they outperformed standard clinicopathological characteristics in predicting LUSC prognosis (Figure 4bc). We discovered that the patient's risk score was inversely proportional to the survival of LUSC patients using a patient's risk survival status plot. Surprisingly, the majority of the novel IncRNAs identified in this research exhibited a negative relationship with our risk model, indicating that more research is needed 
(Figure 4d). For 1, 2, and 3 year survival rates, the AUC predictive value of the unique IncRNAs signature was $0.658,0.693$, and 0.687 , respectively (Figures $4 \mathrm{e}$ ). Risk-related heatmap: ferroptosis-related IncRNAs that such as C10orf55, AC016924.1, AL161431.1, LUCAT1, AC104248.1, MIR3945HG, were highly expressed in a high-risk group, which means all of them might be detrimental to the prognosis of LUSC patients (Figures 4f). COX analysis revealed that IncRNA signature (HR: 1.434, 95Cl: 1.315-1.565), age (HR: 1.022, 95Cl: 1.005-1.039), and tumor stage (HR: 1.242, 95Cl: 1.050-1.470) were mostly independent prognostic variables for LUSC patients' OS (Figure $5 a-b$ ). Figure $5 c$ demonstrates the link between IncRNA and mRNA. The heatmap for the prognosis signature of ferroptosis-related IncRNAs and clinicopathological manifestations was also evaluated (Figure 6). The hybrid nomogram (Figure 7) integrating clinicopathological features and the novel ferroptosis-related IncRNAs prognostic signature was stable and accurate, and hence may be employed in LUSC patient care.

Figure 4. Ferroptosis-related IncRNAs signature. (a) Kaplan-Meier curves result, (b). The AUC values of the risk factors, (c). The DCA of the risk factors. (d). Risk survival status plot, (e). The AUC of the for the prediction of 1, 2, 3-year survival rate of LUSC, (f) Heatmap of different IncRNAs.

Figure 5. COX analysis for the expression of ferroptosis-related IncRNAs, both univariate and multivariate. (a). univariate, (b). multivariate, (c). The relationship between the novel IncRNA and mRNA expression.

Figure 6. Prognostic hallmark and clinicopathological symptoms of ferroptosis-related IncRNAs in a heatmap.

Figure 7: A nomogram for prognostic ferroptosis-related IncRNAs as well as clinic-pathological variables.

\subsection{Gene set enrichment analyses}

According to gene set enrichment analyses (GSEA), the majority of the novel ferroptosis-related IncRNAs prognostic signature regulated immune and tumor-related pathways such as graft versus host disease, allograft rejection, asthma, type i diabetes mellitus, nod like receptor signaling pathway, chemokine signaling pathway, jak stat signaling pathway etc. The top 6 enriched functions or pathways for each cluster are shown, (Figure 8) and (Table S6). FDR q-value and FWER p-value were both $<0.05$. As a consequence, the 'NOD LIKE RECEPTOR SIGNALING PATHWAY' was the most enriched, and some of the genes were positively correlated with $\mathrm{H}$ or $\mathrm{L}$.

Figure 8. Gene set enrichment analyses for ferroptosis-related IncRNAs.

\subsection{Immunity and gene expression}

Figure 9 demonstrates a heatmap of immunological responses generated using the CIBERSORT, ESTIMATE, MCP counter, single-sample gene set enrichment analysis (ssGSEA), and TIMER algorithms. Based on SSGSEA of TCGA-LUSC data, correlation analysis revealed that CCR, Inflammation-promoting, and other immune cell subpopulations and related functions were significantly different between the lowrisk and high-risk groups (Figure 10a). Given checkpoint inhibitor-based immunotherapies are just as important, we investigated into the differences in immune checkpoint expression between the two groups. 
Between the two groups of patients, we discovered a significant discrepancy in the expression of TMIGD2, TNFRSF4, CD244, NRP1, CD276, and other genes (Figure 10b). The expression of YTHDF1, METTL3, FTO, HNRNPC, YTHDC1 were meaningful when ferroptosis-related mRNA expression was compared between the high and low risk groups (Figure 11).

Figure 9. CIBERSORT, ESTIMATE, MCPcounter, SSGSEA, and TIMER algorithms were used to construct a heatmap for immune responses in high and low risk groups.

Figure 10. (a). ssGSEA for the association between immune cell subpopulations and related functions (b). Immune checkpoint expression in high and low LUSC risk groups.

Figure 11.The expression of ferroptosis-related genes in LUSC risk groups with high and low LUSC risk.

\section{Discussion}

Treating LUSC is a complex psychological challenge due to its advanced stage and poor prognosis ${ }^{\square}$. Diagnostic biomarkers and treatment targets for LUSC should constantly be highlighted at the molecular level. According to previous research, ferroptosis is implicated in the pathological cell death associated with degenerative illnesses, and it can also overcome chemotherapy resistance in malignant cells and increase the removal of defective cells ${ }^{[-]}$. Ferroptosis has the potential to operate as a tumor suppressor, making it a viable cancer treatment option ${ }^{\square}$. Despite this, it's unclear how it affects LUSC development through modulating IncRNA. The involvement of immune infiltrating cells in the TME and immune checkpoint inhibitors in the prognosis of LUSC was investigated by this researcher. The findings of this study resulted in the discovery of a promising biomarker and therapeutic target.

We retrieved ferroptosis-related gene expression data and differentiated between mRNA and IncRNA in this study. Co-expression analysis was utilized to look at the relationship between ferroptosis-related gene expression and IncRNAs. Using the co-expression network plot, we observed phenomena in which numerous IncRNAs were associated with ferroptosis-related genes in LUSC. After that, we discovered 102 DEGs associated with ferroptosis. KEGG analyses further revealed the genes mainly participated in Chemical carcinogenesis-reactive oxygen species, Ferroptosis, MicroRNAs in cancer, HIF-1 signaling pathway, NOD-like receptor signaling pathway. A growing body of research reveals that miRNA and IncRNA are important regulators of ferroptosis. By reducing iron absorption, Nrf2 lowers the production of reactive oxygen species (ROS). As a result, miRNA inhibits ferroptosis via regulating the expression of Nrf2. Meanwhile, miRNA is engaged in iron transport, storage, usage, and absorption control ${ }^{[-]}$. The interplay of MTOR and GPX4 signaling regulates autophagy-dependent ferroptotic cancer cell death ${ }^{\square}$. HIF$1 \mathrm{a}$ is highly expressed in cancer-associated fibroblasts (CAFs), and HIF-1a-expressed fibroblasts activate the NF-KB signaling pathway, which promotes lung cancer tumor growth ${ }^{\square}$. Yana Zhang ${ }^{\square}$ believes that HIF$1 \mathrm{a}$ is a critical factor of CAFs in lung cancer, and that targeting HIF-1a-expressed CAFs could be a future anticancer treatment. Visibility. In LUSC, ferroptosis is crucial. 
To study their possible activities in LUSC, the ferroptosis-associated IncRNAs were split into two categories: high-risk and low-risk. Using data on prognosis-related IncRNAs, the confidence interval and the hazard ratio were calculated. In a university Cox regression study, ferroptosis-related IncRNAs appeared to be strongly correlated with LUSC prognosis. This study identified eight ferroptosis-related IncRNAs that have been linked to prognosis and show altered expression in high-risk and low-risk patients. Some IncRNAs were considered to be overexpressed in high-risk people, while others were found to be overexpressed in low-risk people $(\mathrm{P}<0.05)$. We looked into the role of ferroptosis-related IncRNAs in LUSC in more detail. The predictive value of ferroptosis-related IncRNAs was measured using a survival analysis based on IncRNA subtypes. Low-risk IncRNAs were involved in a better prognosis than high-risk IncRNAs. According to the ferroptosis-related IncRNAs risk score, C10orf55, AC016924.1, AL161431.1, LUCAT1, AC104248.1, and MIR3945HG were significantly expressed in the high-risk group, demonstrating that they are LUSC oncogenes. Furthermore, the above-mentioned ferroptosis-related IncRNAs could be invoked as a therapeutic target for LUSC. In the LUSC study, IncRNAs were also related to patient outcomes. Only a little amount of study has been done on IncRNA changes connected to ferroptosis. More research is needed in order to fully understand the process of ferroptosis-related IncRNA modification and identification, as well as to corroborate our findings.

We also investigated at and computed the infiltration of different immune cells in the samples to find out what function immune cell infiltration and the TME play in LUSC. CCR, HLA, Inflammation-promoting, Parainflammation, T cell co-inhibition, T cell co-stimulation, Type I IFN Reponse, Type II IFN Reponse considerably infiltrated tumor tissues in high-risk individuals, according to research of immune cell infiltration discrepancies. As a result, these cells' invasion of the TME may have a negative effect on LUSC patients' prognosis. Ferroptosis and immune checkpoint inhibitors (ICls) work together to improve anticancer efficacy in ICl-resistant cancers ${ }^{\square}$. TMIGD2, TNFRSF4, CD244, NRP1, CD276, ICOS, CD80, and other checkpoint genes were considered to be highly expressed in our study, suggesting that they could become ICls in LUSC. The relationship between ICl and ferroptosis has received very little attention. P53, ATF3/4, SLC7A11, ACSL4, and the BECN1 pathway are among the latest ferroptosis-regulating factors found in recent years. Surprisingly, IncRNA is connected to the regulation of these factors' expression $\square$. Despite the fact that research on ferroptosis-related InRNA and LUSC is limited. We might conclude that changes in ferroptosis-related IncRNAs are associated with the onset and progression of LUSC based on the information presented above.

In GSEA, the nod like receptor-signaling pathway was found to be the most significantly enriched pathway. NOD-like receptors are involved in inflammatory responses that exacerbate the occurrence and development of lung remodeling. when there is a hypoxia plateau ${ }^{\square}$. Ferroptosis-related IncRNAs may regulate LUSC cell migration and proliferation through modulating the NOD LIKE RECEPTOR SIGNALING PATHWAY, based on the aforementioned properties. The low-risk subtype surpassed the high-risk subtype in terms of survival. The low-risk subtype showed a high survival rate than the high-risk subtype, according to the ferroptosis-related IncRNA prognostic model. Furthermore, our model is under a high level of accuracy when it comes to forecasting LUSC patient survival. Increases in risk score are 
connected to higher death rates and a higher high-risk ratio. Our model had no effect on other clinical prognostic variables that could influence patient outcomes. The principle could be applicable to a variety of clinical situations. Ferroptosis-related IncRNAs seem to be viable biomarkers for predicting LUSC patient outcomes, based on our findings and data from the literature.

Even while our research provides some theoretical foundations and research recommendations, there are still limitations. Using the TCGA dataset, we first constructed and validated a ferroptosis-related IncRNA prediction signature. We were unable to obtain sufficient external data from other public sources in order to evaluate the model's reliability. Second, only the signature's eight ferroptosis-related IncRNAs were subjected to preliminary expression studies. Regardless, no more functional or mechanistic research was conducted. Finally, no studies in LUSC have been undertaken to confirm the relationship between prognostic IncRNAs and ferroptosis. However, we shall conduct extra investigation in order to completely appreciate the aforementioned facts.

\section{Conclusions}

In conclusion, we looked for prognosis-related ferroptosis-related IncRNAs by analyzing the expression patterns and clinical data of LUSC samples from the TCGA database. As part of the ferroptosis regulation, 8 ferroptosis-related predictive IncRNAs were discovered in 502 LUSC patients. For LUSC, it has a significant predictive value. Our findings contribute to the understanding of ferroptosis-related IncRNAs and immune cell infiltration in the TME, possibly paving the way for novel therapeutic targets and prognostic indicators in the future. It is desirable for our findings will benefit identify ferroptosisrelated IncRNA that stimulates LUSC growth, allowing us to understand more about their possible function in the development and progression of LUSC tumors.

\section{Abbreviations}

\begin{tabular}{|llll|}
\hline LUSC & Lung squamous cell carcinoma & TCGA & The Cancer Genome Atlas \\
\hline GO & Gene Ontology & IncRNAs & long non-coding RNAs \\
\hline AUC & areas under the curve & BP & Biological processes \\
\hline MF & molecular functions & CC & cellular components \\
\hline KEGG & Kyoto Encyclopedia of Genes and Genomes & DEGs & differentially expressed genes \\
\hline ROC & receiver-operating characteristics & DCA & Decision Curve Analysis \\
\hline GSEA & gene set enrichment analyses & TME & tumor microenvironment \\
\hline ICls & immune checkpoint inhibitors & OS & overall survival \\
\hline
\end{tabular}

\section{Declarations}




\section{Data availability}

Patients who have provided informed consent for the use of their data have been included in the TCGA database, which is a public database. Users can freely obtain and publish appropriate articles based on the relevant data. Our study has no ethical difficulties or conflicts of interest because it is built on opensource data.

\section{Ethics approval and consent to participation}

This manuscript is not a clinical trial, hence the ethics approval and consent to participation is not applicable.

\section{Consent for publication}

All authors have read and approved this manuscript to be considered for publication.

\section{Competing interests}

The authors declare no competing financial interests.

\section{Acknowledgements}

Thanks to professor Huang for his strict guidance on this paper, and thanks to Miss Huang and Miss Cai of support for this paper. Thanks to reviewers and editors for their sincere comments.

\section{Fund}

1. National Natural Science Foundation of China (NSFC), 82160938, "The effect of TGF- $\beta$ 1-mediated ERK and Smad signaling pathway on the anti-renal interstitial fibrosis mechanism of drug-cake moxibustion"; 2. Health And Health Commission of Yunnan Province 2020 High-level TCM Reserve Talents (Acupuncture treatment of chronic kidney disease) Incubation project (Yunwei TCM Development Development [2021] No. 1) 3. The second round of construction project of The National Traditional Chinese Medicine School Heritage Studio of the State Administration of Traditional Chinese Medicine (National Traditional Chinese Medicine Teaching Letter [2019] 62); 4. Yunnan Provincial Health Commission "2020 High-level Traditional Chinese Medicine Talents Training Target" project (Yunnan Traditional Chinese Medicine Development (2021) No. 1); 5. Yunnan Provincial Department of Science and Technology-Joint Special General Project of Traditional Chinese Medicine (No.2019FF002-022); 6. Social Science Project of Yunnan University of Traditional Chinese Medicine (No. [2020] -SkyB-00016).

\section{Author Contributions}

Zixuan Wu drafted and revised the manuscript. Xuyan Huang and Minjie Cai are in charge of data collection. Peidong Huang conceived and designed this article, in charge of syntax modification and 
revise of the manuscript. Zunhui Guan revised the manuscript. All the authors have read and agreed to the final version manuscript.

\section{References}

1. Nasim F,Sabath BF,Eapen GA. Lung Cancer. Med Clin North Am. 2019 May;103(3):463-473.

2. Gavin S Jones,David R Baldwin. Recent advances in the management of lung cancer[J]. Clinical Medicine,2018,18(Suppl 2):

3. Schabath Matthew B,Cote Michele L. Cancer Progress and Priorities: Lung Cancer.[J]. Cancer epidemiology, biomarkers \& prevention : a publication of the American Association for Cancer Research, cosponsored by the American Society of Preventive Oncology,2019,28(10):

4. Roberto Ruiz-Cordero,Walter Patrick Devine. Targeted Therapy and Checkpoint Immunotherapy in Lung Cancer[J]. Surgical Pathology Clinics,2020,13(1):

5. Wang Rui,Xing Rui,Su Qi,et al. Knockdown of SFRS9 Inhibits Progression of Colorectal Cancer Through Triggering Ferroptosis Mediated by GPX4 Reduction [J]. Frontiers in Oncology,2021:

6. Chaudhary Nazia,Choudhary Bhagya Shree,Shah Sanket Girish,et al. Lipocalin 2 expression promotes tumor progression and therapy resistance by inhibiting ferroptosis in colorectal cancer[J]. International Journal of Cancer,2021,149(7):

7. Liu Kai,Chen Shaoxi,Lu Ruoyi. Identification of Important Genes Related to Ferroptosis and Hypoxia in Acute Myocardial Infarction Based on WGCNA.[J]. Bioengineered,2021:

8. Dietrich Cornelia,Hofmann Thomas G.. Ferroptosis Meets Cell-Cell Contacts[J]. Cells,2021,10(9):

9. Xu Shan,Min Junxia,Wang Fudi. Ferroptosis: an emerging player in immune cells[J]. Science Bulletin,2021,66(22):

10. Wu Chenyao,Liu Zhonglong,Chen Zhixin,et al. A nonferrous ferroptosis-like strategy for antioxidant inhibition-synergized nanocatalytic tumor therapeutics.[J]. Science advances,2021,7(39):

11. Emmons Michael F,Smalley Keiran S M. Ironing-Out the Details: New Strategies for Combining Ferroptosis Inhibitors with Immunotherapy in Melanoma.[J]. The Journal of investigative dermatology,2021:

12. Liu Mingcheng,Wang Lirong,Xia Xiaojing,et al. Regulated lytic cell death in breast cancer.[J]. Cell biology international,2021:

13. Zhao Enfa,Chen Shimin,Dang Ying. Development and External Validation of a Novel Immune Checkpoint-Related Gene Signature for Prediction of Overall Survival in Hepatocellular Carcinoma.[J]. Frontiers in molecular biosciences,2020,7:

14. Wang Zhining,Jensen Mark A,Zenklusen Jean Claude. A Practical Guide to The Cancer Genome Atlas (TCGA).[J]. Methods in molecular biology (Clifton, N.J.),2016,1418:

15. Zhou Nan,Bao Jinku. FerrDb: a manually curated resource for regulators and markers of ferroptosis and ferroptosis-disease associations.[J]. Database : the journal of biological databases and curation,2020,2020: 
16. Newman Aaron M,Liu Chih Long,Green Michael R,et al. Robust enumeration of cell subsets from tissue expression profiles.[J]. Nature methods,2015,12(5):

17. Pornpimol Charoentong,Francesca Finotello,Mihaela Angelova,et al. Pan-cancer Immunogenomic Analyses Reveal Genotype-Immunophenotype Relationships and Predictors of Response to Checkpoint Blockade[J]. Cell Reports,2017,18(1):

18. Kosuke Yoshihara,Maria Shahmoradgoli,Emmanuel Martínez,et al. Inferring tumour purity and stromal and immune cell admixture from expression data[J]. Nature Communications,2013,4(1):

19. Shi Jingqi,Jiang Dongbo,Yang Shuya,et al. LPAR1, Correlated With Immune Infiltrates, Is a Potential Prognostic Biomarker in Prostate Cancer[J]. Frontiers in Oncology,2020:

20. Yi Ming,Nissley Dwight V,McCormick Frank,et al. ssGSEA score-based Ras dependency indexes derived from gene expression data reveal potential Ras addiction mechanisms with possible clinical implications.[J]. Scientific reports,2020,10(1):

21. Li Taiwen,Fan Jingyu,Wang Binbin,et al. TIMER: A Web Server for Comprehensive Analysis of TumorInfiltrating Immune Cells.[J]. Cancer research,2017,77(21):

22.

23. Wan Seok Yang,Brent R. Stockwell. Ferroptosis: Death by Lipid Peroxidation[J]. Trends in Cell Biology,2016,26(3):

24. Jose Pedro Friedmann Angeli,Manuela Schneider,Bettina Proneth,et al. Inactivation of the ferroptosis regulator Gpx4 triggers acute renal failure in mice[J]. Nature Cell Biology,2014,16(12):

25. Brent R. Stockwell,José Pedro Friedmann Angeli,Hülya Bayir,Ashley I. Bush,et al. Ferroptosis: A Regulated Cell Death Nexus Linking Metabolism, Redox Biology, and Disease[J]. Cell,2017,171(2):

26. Kabaria Savan,Choi Doo Chul,Chaudhuri Amrita Datta,et al. MicroRNA-7 activates Nrf2 pathway by targeting Keap1 expression.[J]. Free radical biology \& medicine,2015,89:

27. Muhua Yang,Yuan Yao,Gabriel Eades,et al. MiR-28 regulates Nrf2 expression through a Keap1independent mechanism[J]. Breast Cancer Research and Treatment,2011,129(3):

28. Xu Dawei,Zhu Hao,Wang Chengniu,et al. microRNA-455 targets cullin 3 to activate Nrf2 signaling and protect human osteoblasts from hydrogen peroxide.[J]. Oncotarget,2017,8(35):

29. Liu Yang,Wang Yuan,Liu Jiao,et al. Interplay between MTOR and GPX4 signaling modulates autophagy-dependent ferroptotic cancer cell death.[J]. Cancer gene therapy,2021,28(1-2):

30. Elyada Ela,Bolisetty Mohan,Laise Pasquale,et al. Cross-Species Single-Cell Analysis of Pancreatic Ductal Adenocarcinoma Reveals Antigen-Presenting Cancer-Associated Fibroblasts.[J]. Cancer discovery,2019,9(8):

31. Zhang Y,Bian Y,Wang Y, et al. HIF-1a is necessary for activation and tumour-promotion effect of cancer-associated fibroblasts in lung cancer. J Cell Mol Med. 2021 Jun;25(12):5457-5469.

32. Rong Tang,Jin Xu,Bo Zhang,et al. Ferroptosis, necroptosis, and pyroptosis in anticancer immunity[J]. Journal of Hematology \& Oncology,2020,13(1): 
33. Song Xiaohua,Long Dingxin. Nrf2 and Ferroptosis: A New Research Direction for Neurodegenerative Diseases.[J]. Frontiers in neuroscience,2020,14:

34. Wang $\mathrm{H}$,Lin X,Pu X. NOD-like receptors mediate inflammatory lung injury during plateau hypoxia exposure. J Physiol Anthropol. 2020 Oct 7;39(1):32.

\section{Figures}

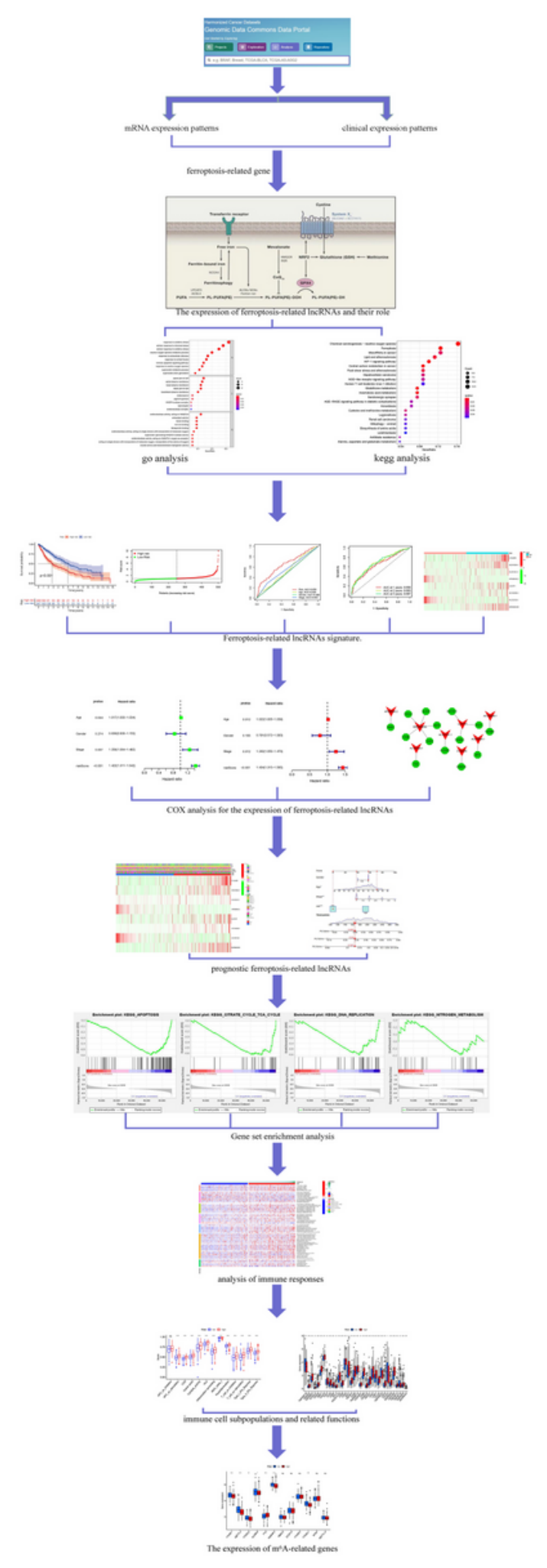


Figure 1

Framework based on an integration strategy of ferroptosis-related IncRNAs.

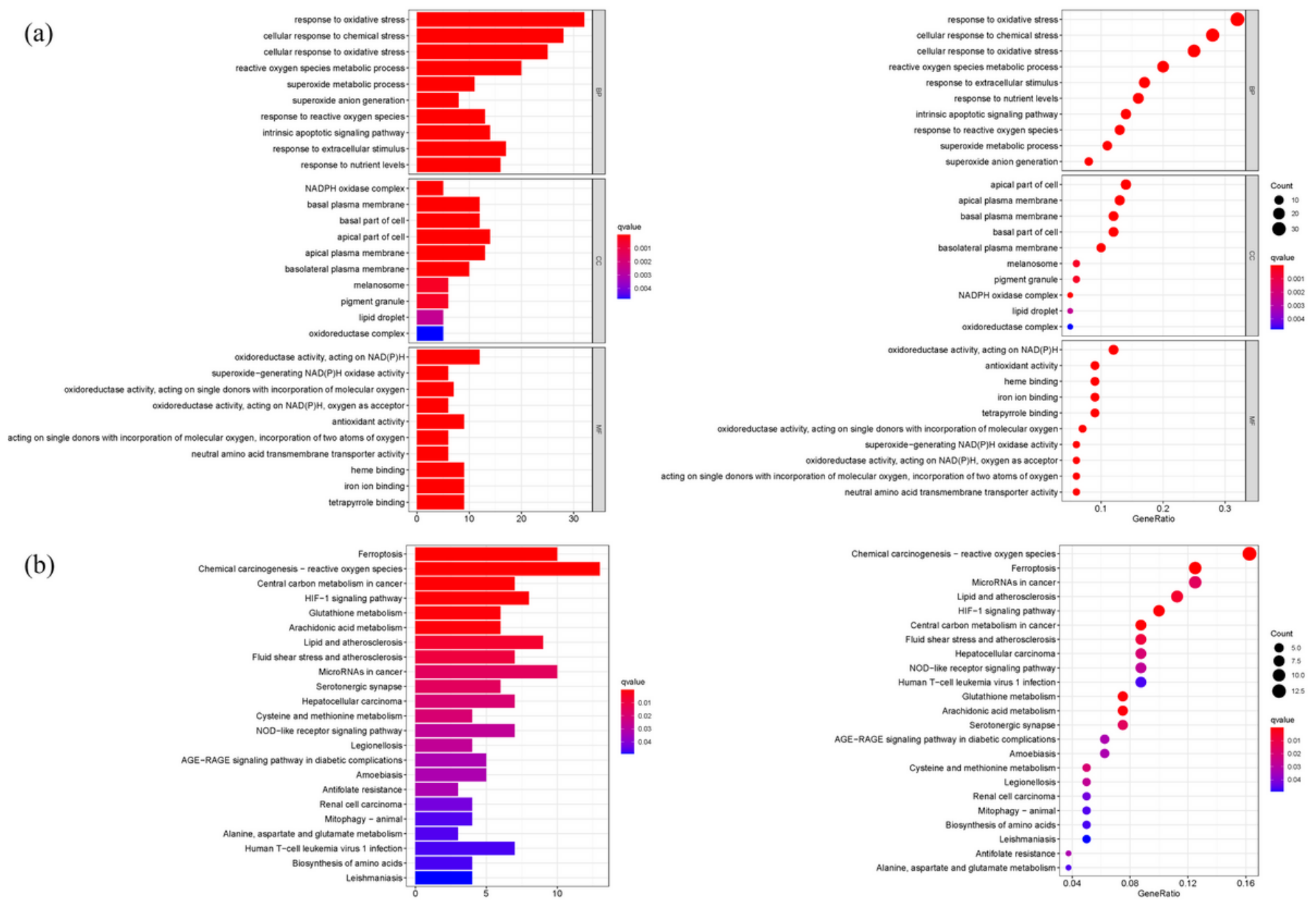

Figure 2

GO and KEGG analyses for ferroptosis-related differentially expressed genes. (a) GO (b) KEGG. 


$\begin{array}{lrr} & \text { pvalue } & \begin{array}{r}\text { Hazard ratio } \\ \text { C10orf55 }\end{array} \\ \text { MIR22HG } & 0.001 & 1.330(1.166-1.517) \\ \text { LINC01322 } & 0.008 & 1.037(1.009-1.067) \\ \text { AC016924.1 } & 0.002 & 1.186(1.045-1.345) \\ \text { AC011511.5 } & 0.022 & 1.041(1.298-3.208) \\ \text { AL136369.1 } & 0.035 & 1.521(1.030-2.247) \\ \text { AL161431.1 } & 0.040 & 1.005(1.000-1.009) \\ \text { AC007823.1 } & 0.045 & 0.652(0.429-0.991) \\ \text { LINC02345 } & 0.034 & 1.193(1.014-1.404) \\ \text { LINC02178 } & 0.044 & 1.016(1.000-1.031) \\ \text { SFTA1P } & 0.030 & 1.015(1.001-1.028) \\ \text { AL606469.1 } & 0.011 & 1.595(1.111-2.289) \\ \text { LRRK2-DT } & 0.047 & 1.097(1.001-1.202) \\ \text { LANCL1-AS1 } & 0.023 & 1.822(1.088-3.054) \\ \text { AP006545.2 } & 0.015 & 0.713(0.543-0.938) \\ \text { LUCAT1 } & 0.015 & 1.105(1.020-1.197) \\ \text { MYOSLID } & 0.029 & 1.038(1.004-1.073) \\ \text { ARHGEF2-AS1 } & 0.041 & 1.663(1.020-2.713) \\ \text { AC104248.1 } & 0.013 & 1.179(1.036-1.342) \\ \text { AL357054.4 } & 0.042 & 1.406(1.012-1.953) \\ \text { AL122125.1 } & 0.020 & 0.761(0.605-0.958) \\ \text { AP001189.1 } & 0.025 & 1.415(1.045-1.915) \\ \text { AP001189.3 } & 0.005 & 1.387(1.105-1.742) \\ \text { LASTR } & 0.006 & 1.034(1.010-1.059) \\ \text { LINC02555 } & 0.006 & 1.438(1.108-1.867) \\ \text { MIR3945HG } & <0.001 & 1.634(1.251-2.135) \\ \text { AL138756.1 } & 0.048 & 1.434(1.002-2.052) \\ \text { MIR762HG } & 0.029 & 0.708(0.520-0.965) \\ \text { AC019080.1 } & 0.038 & 0.932(0.871-0.996)\end{array}$

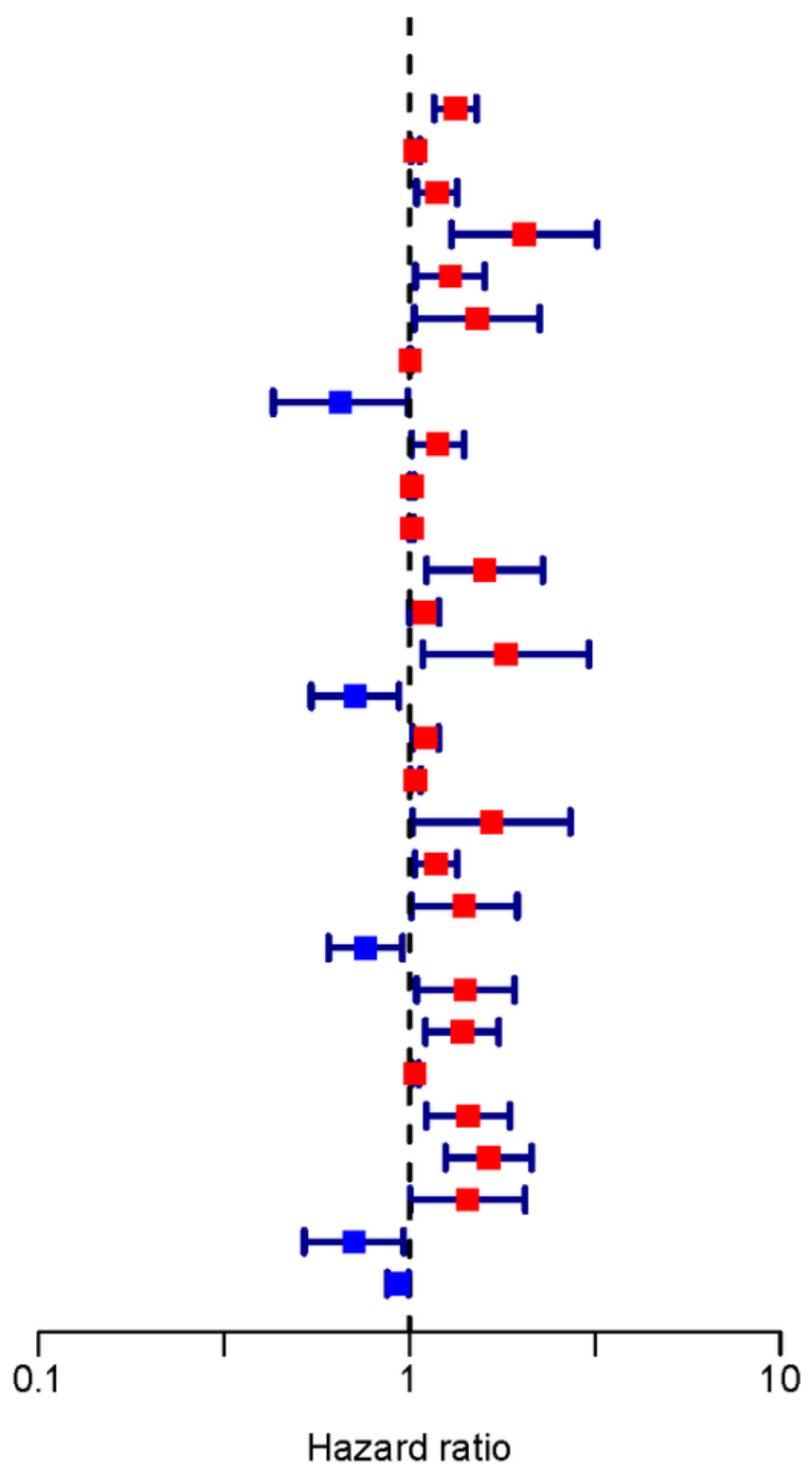

Figure 3

Forest plot of significant IncRNAs. 
(a)

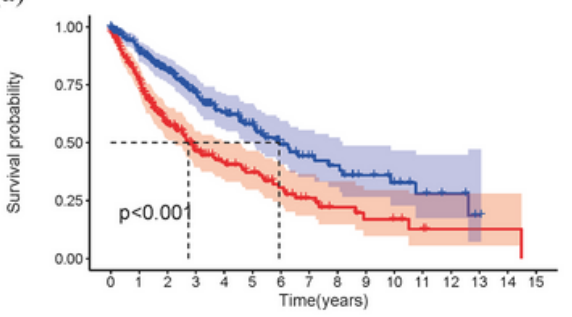

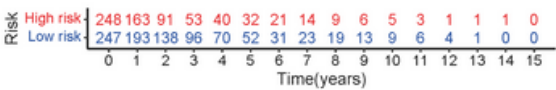

(d)
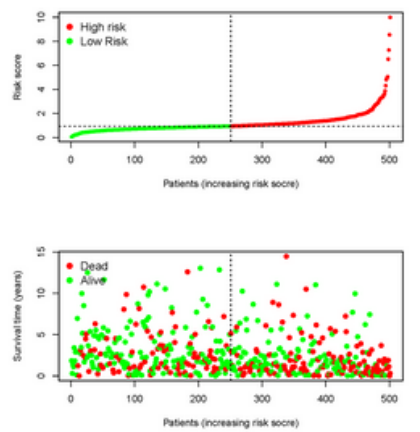

(b)

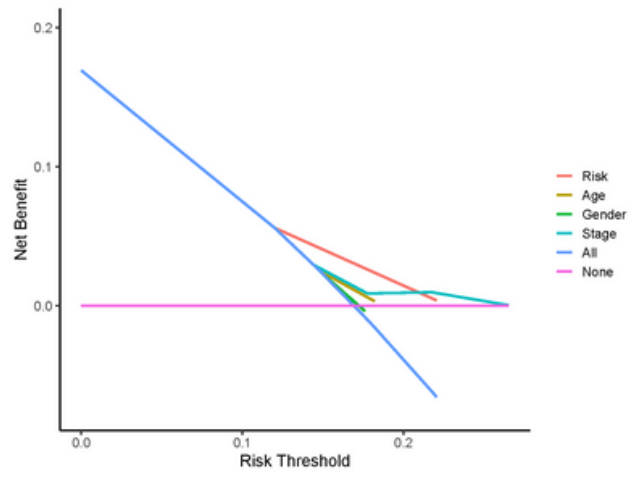

(f)

f) (c)

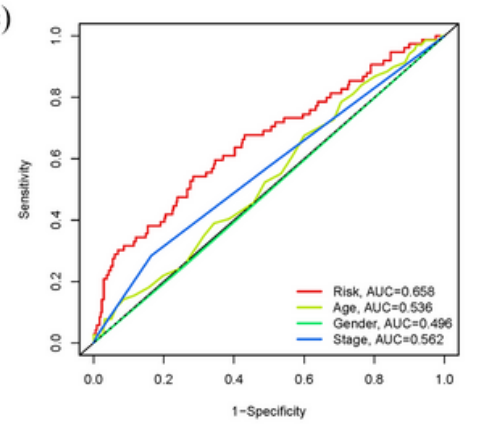

(e)

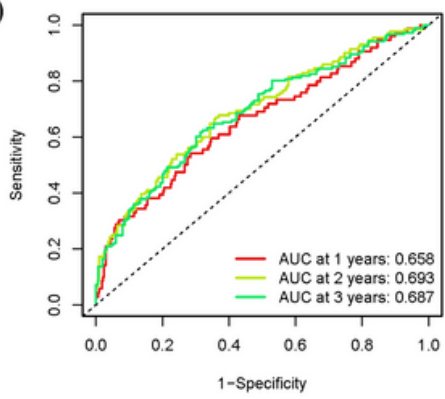

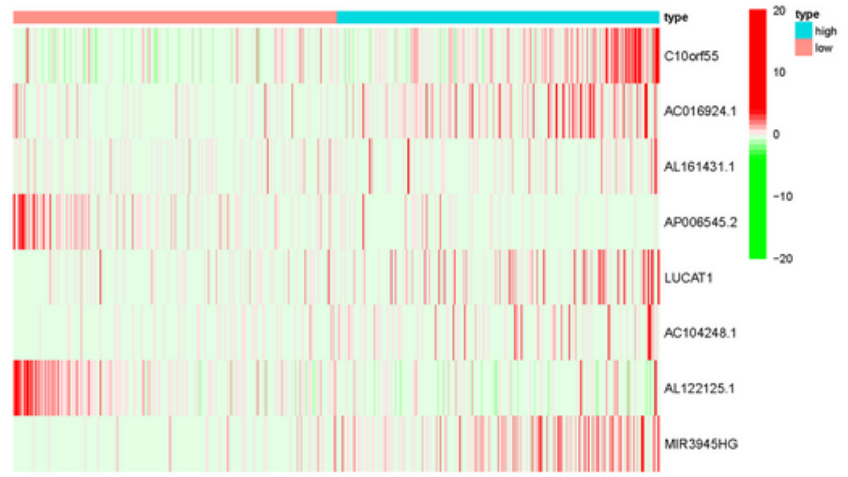

Figure 4

Ferroptosis-related IncRNAs signature. (a) Kaplan-Meier curves result, (b). The AUC values of the risk factors, (c). The DCA of the risk factors. (d). Risk survival status plot, (e). The AUC of the for the prediction of 1, 2, 3-year survival rate of LUSC, (f) Heatmap of different IncRNAs.

(a)

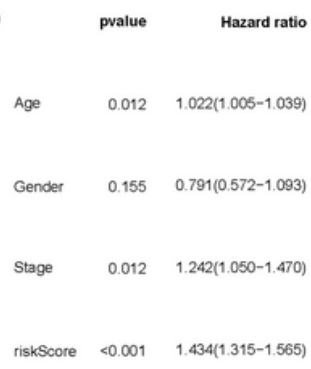

riskScore $<0.001 \quad 1.434(1.315-1.565$

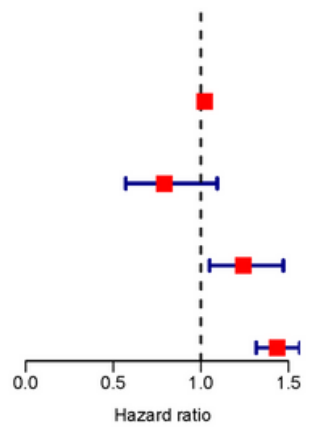

(b)

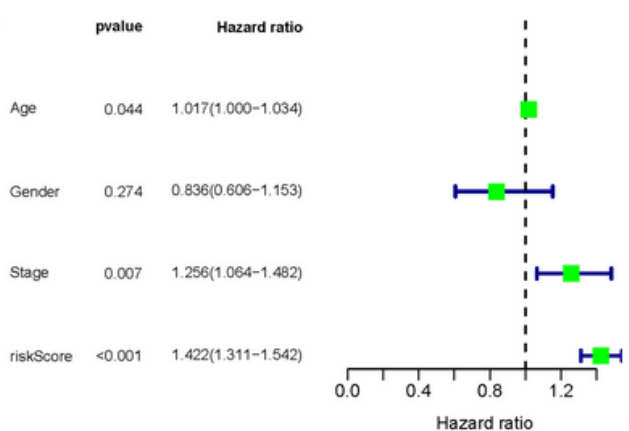

(c)
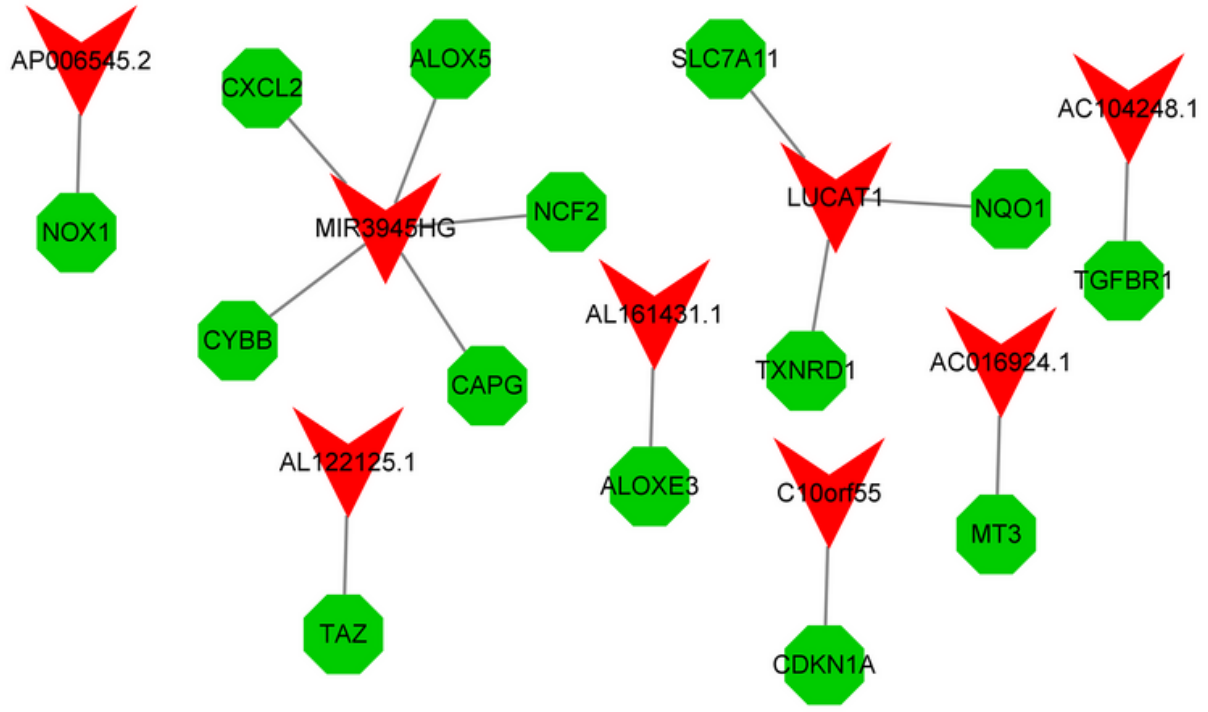

Figure 5 
COX analysis for the expression of ferroptosis-related IncRNAs, both univariate and multivariate. (a). univariate, (b). multivariate, (c). The relationship between the novel IncRNA and mRNA expression.

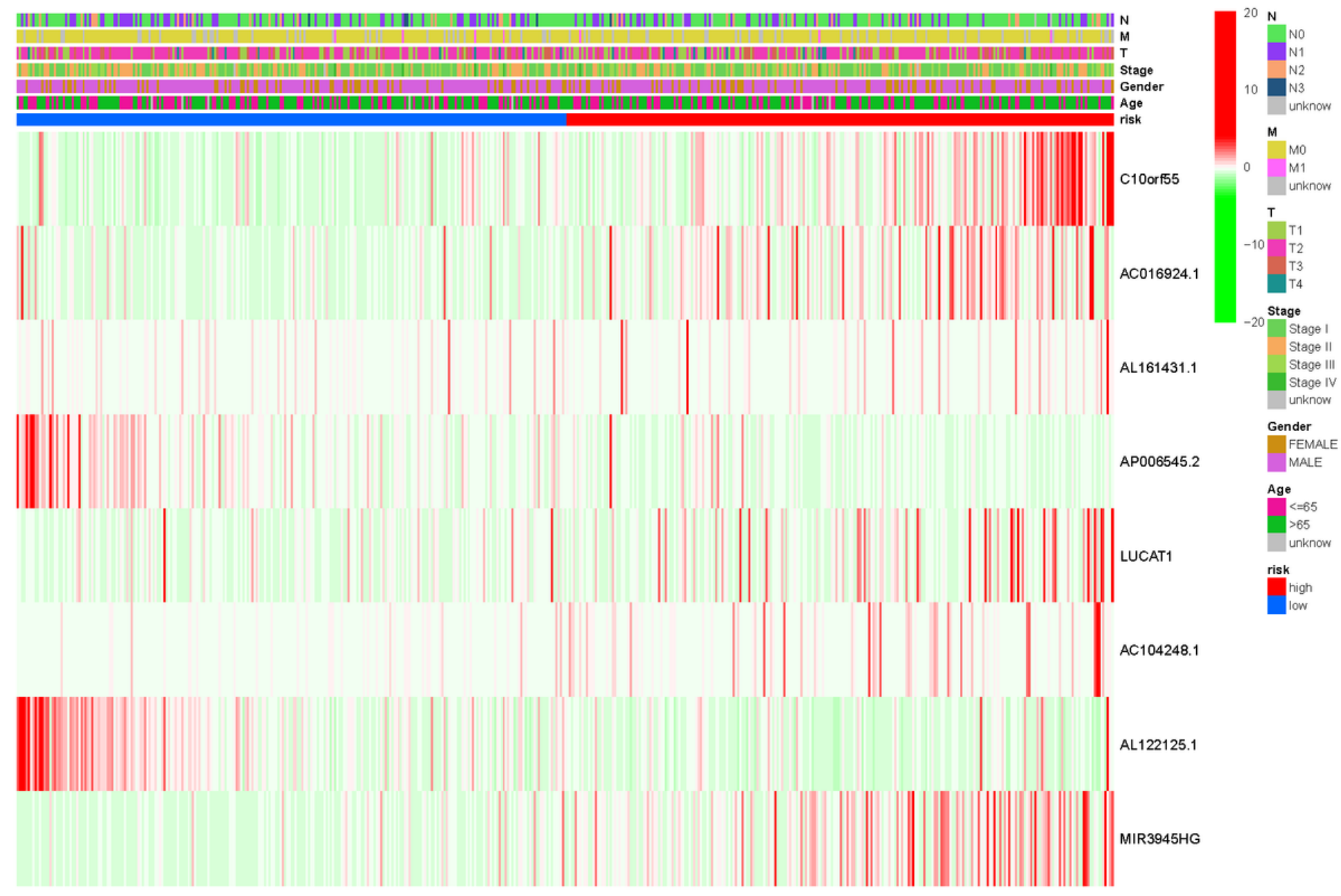

Figure 6

Prognostic hallmark and clinicopathological symptoms of ferroptosis-related IncRNAs in a heatmap. 

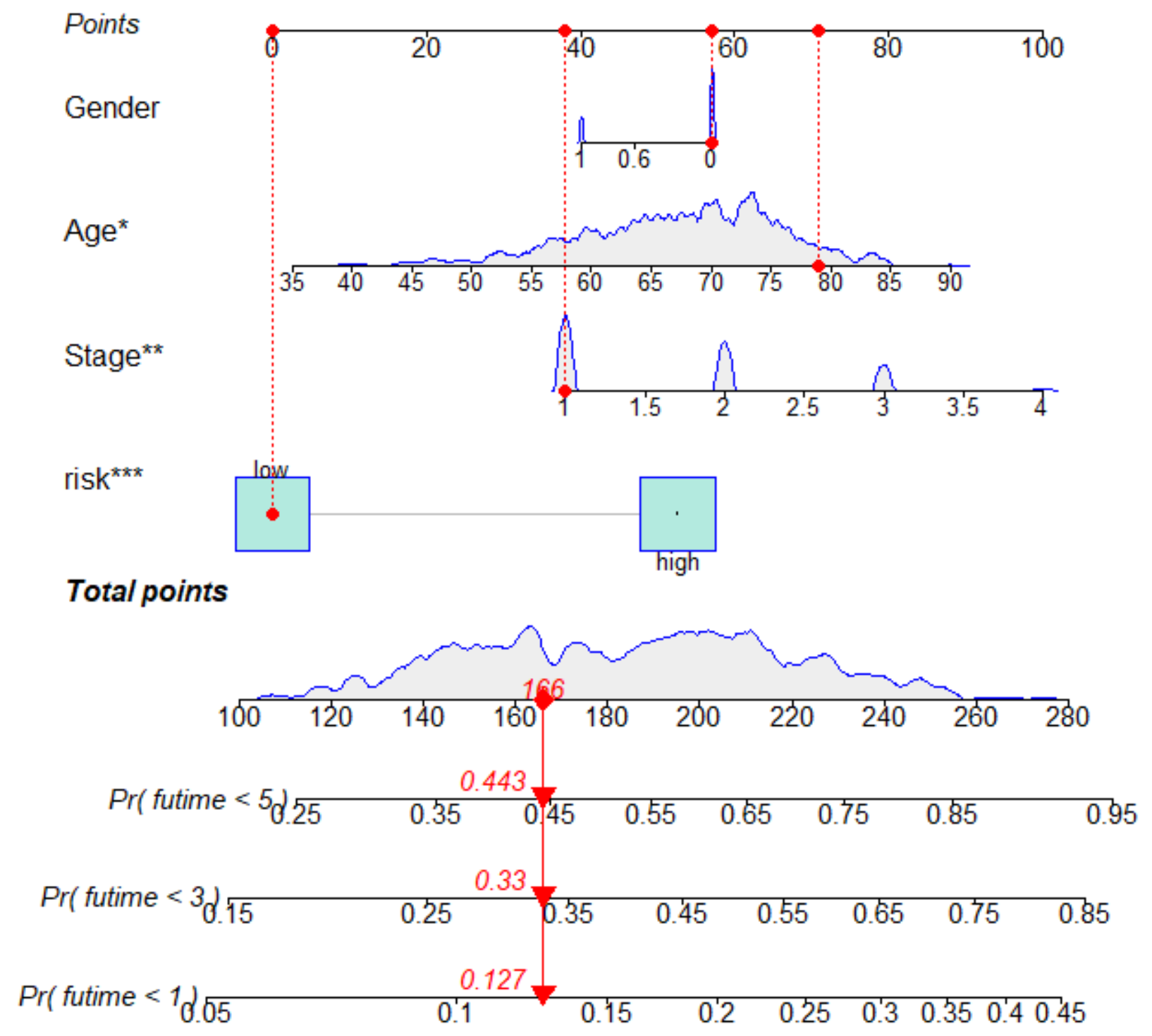

Figure 7

A nomogram for prognostic ferroptosis-related IncRNAs as well as clinic-pathological variables. 

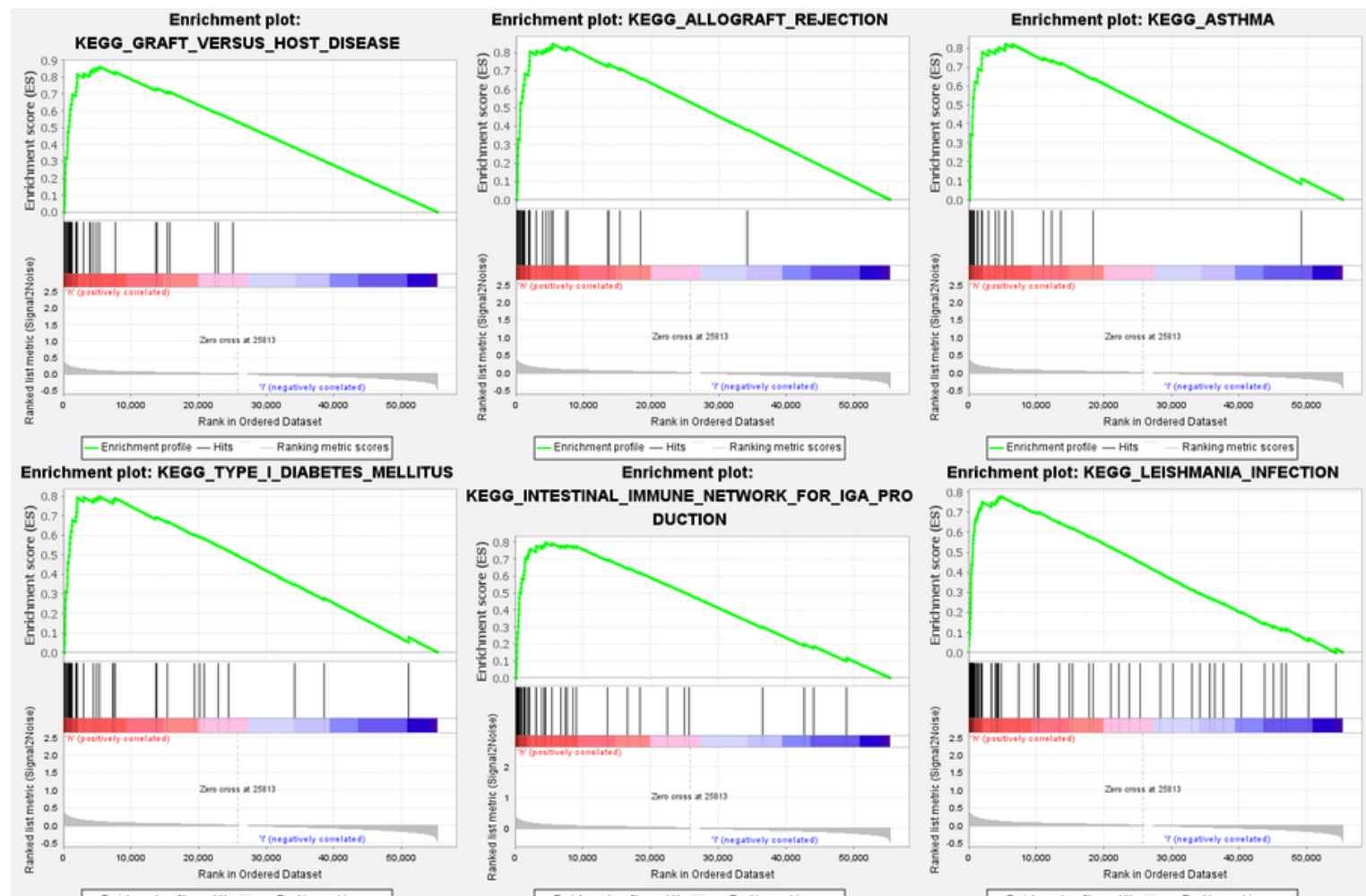

Enrichment plot: KEGG_LEISHMANIA_INFECTION
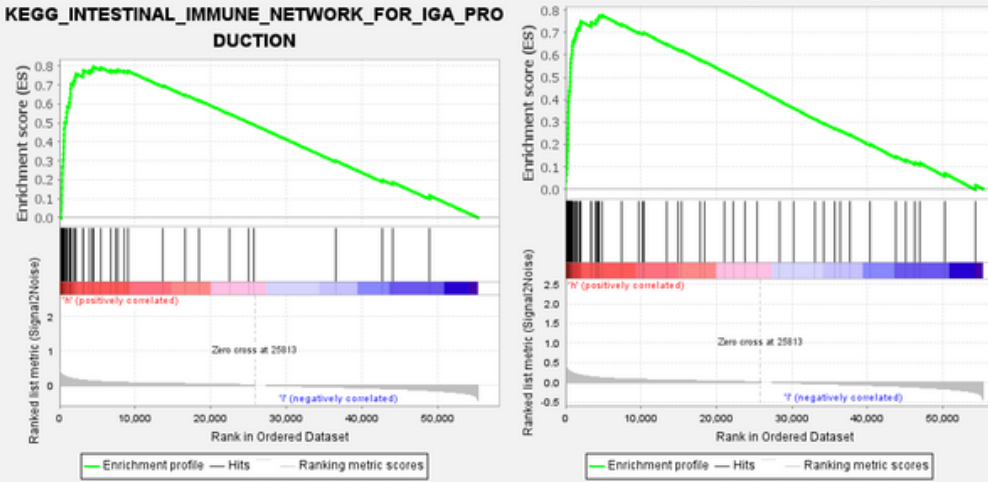

Ranving mettic scores

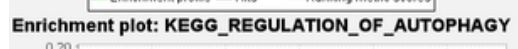

Enrichment plot: KEGG_PEROXISOME
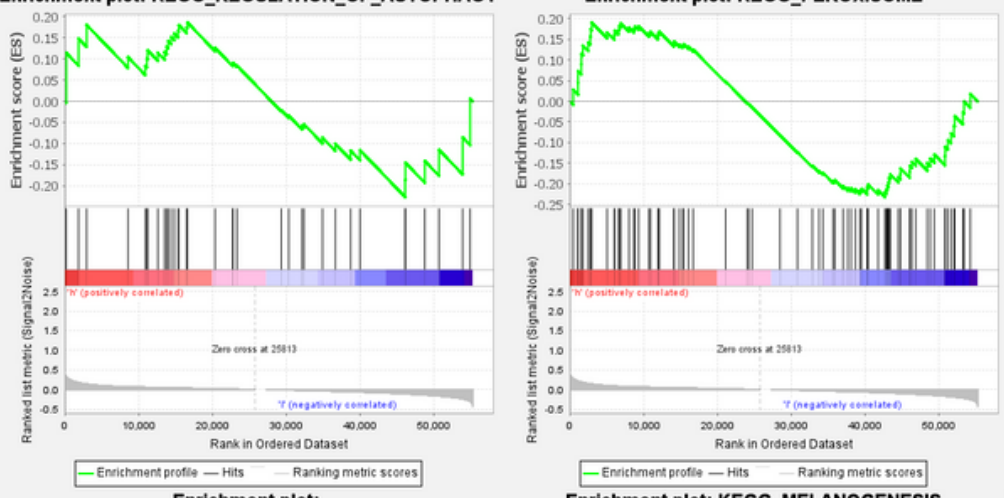

KEGG_STEROID_HORMONE_BIOSYNTHESIS
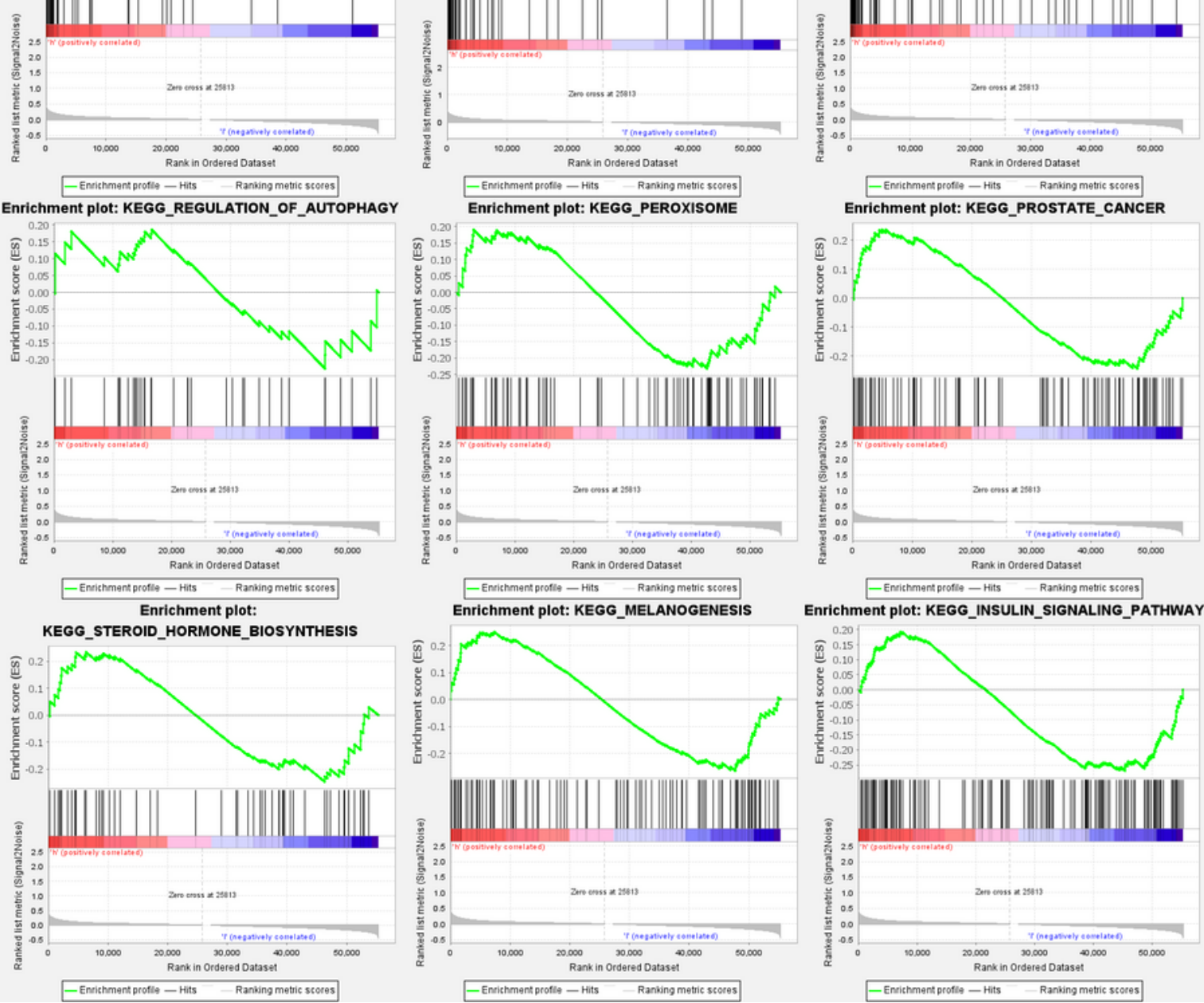

Figure 8

Gene set enrichment analyses for ferroptosis-related IncRNAs. 


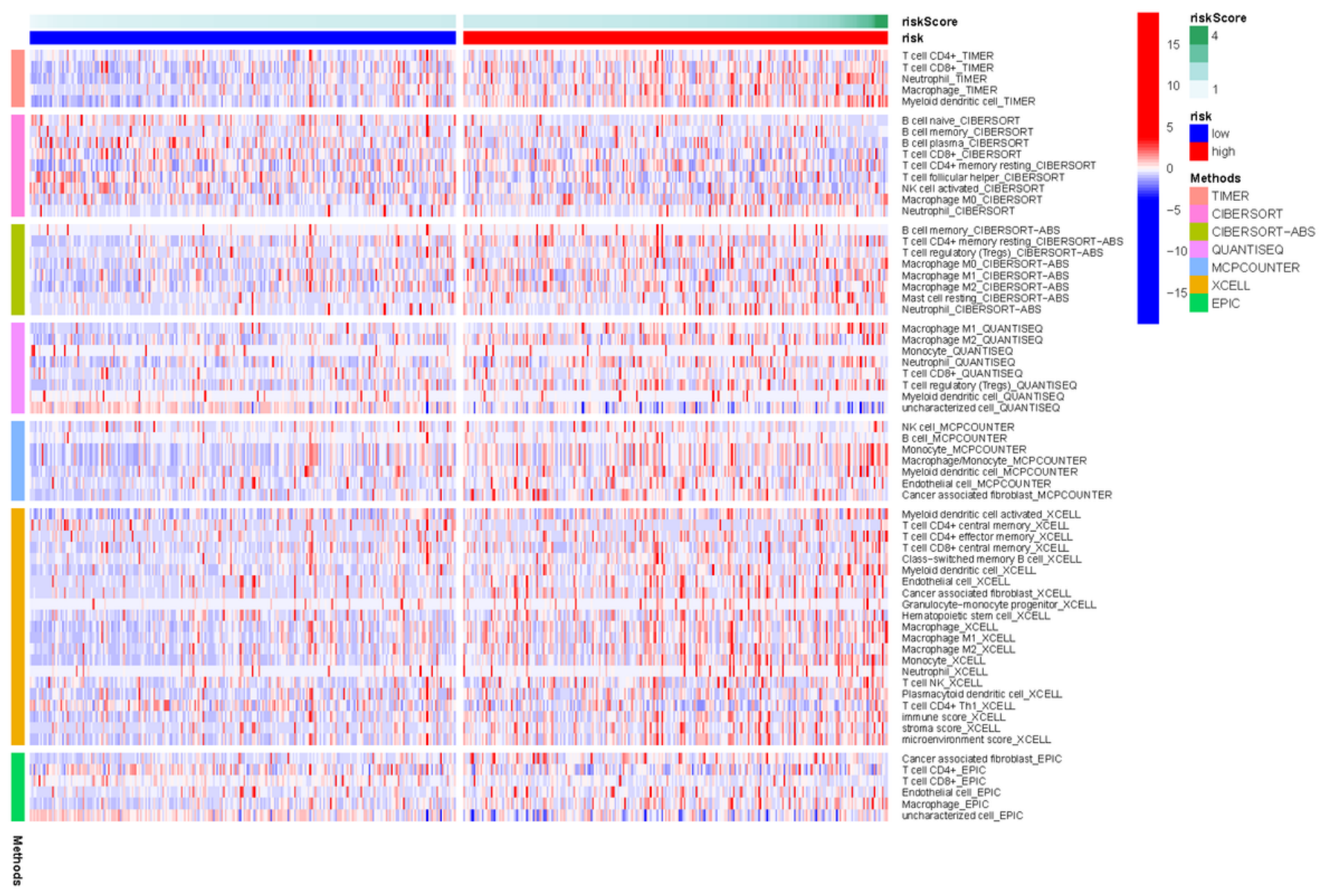

Figure 9

CIBERSORT, ESTIMATE, MCPcounter, ssGSEA, and TIMER algorithms were used to construct a heatmap for immune responses in high and low risk groups.

(a)

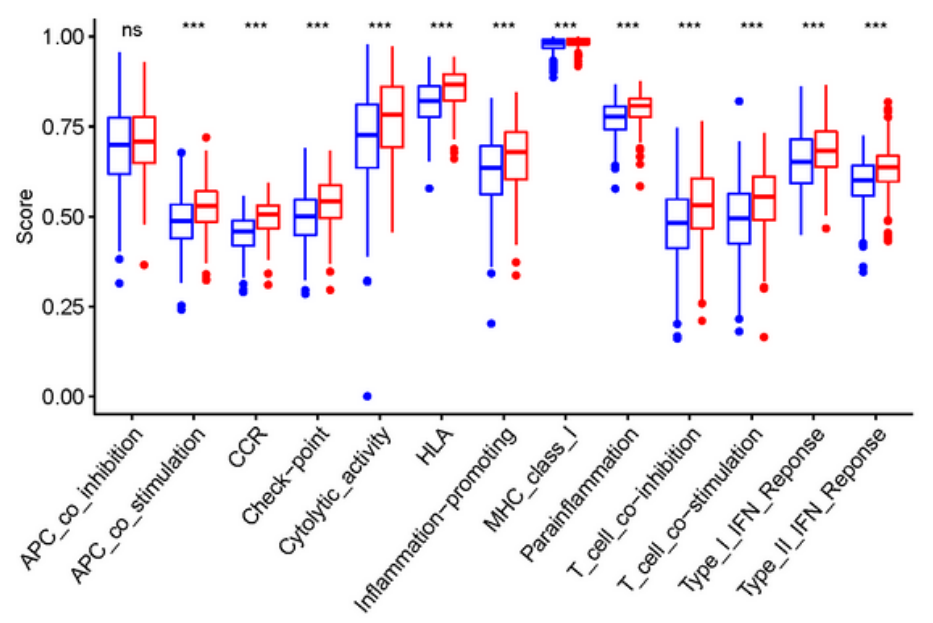

(b)

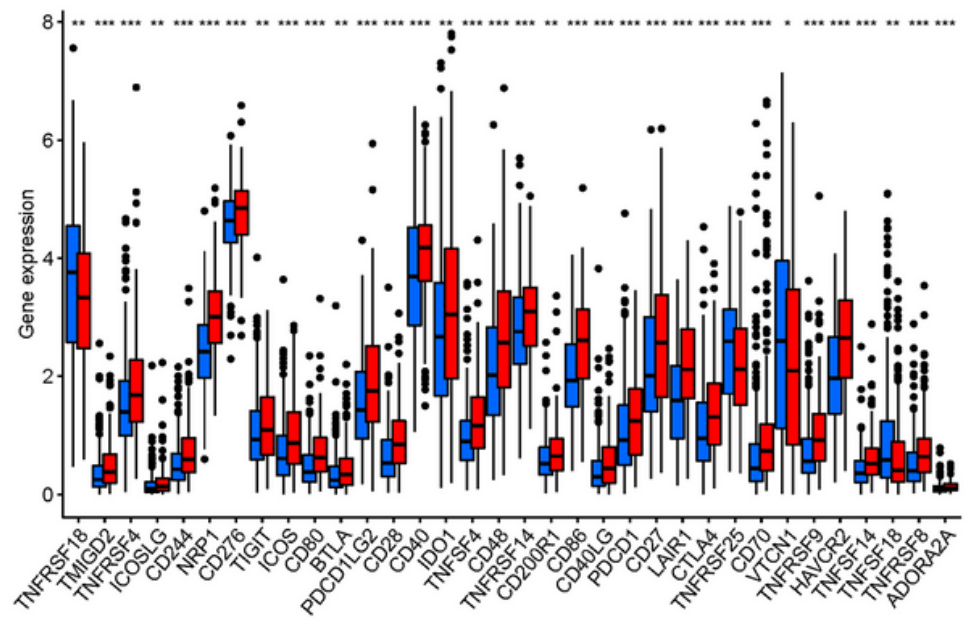

Figure 10 
(a). SSGSEA for the association between immune cell subpopulations and related functions (b). Immune checkpoint expression in high and low LUSC risk groups.

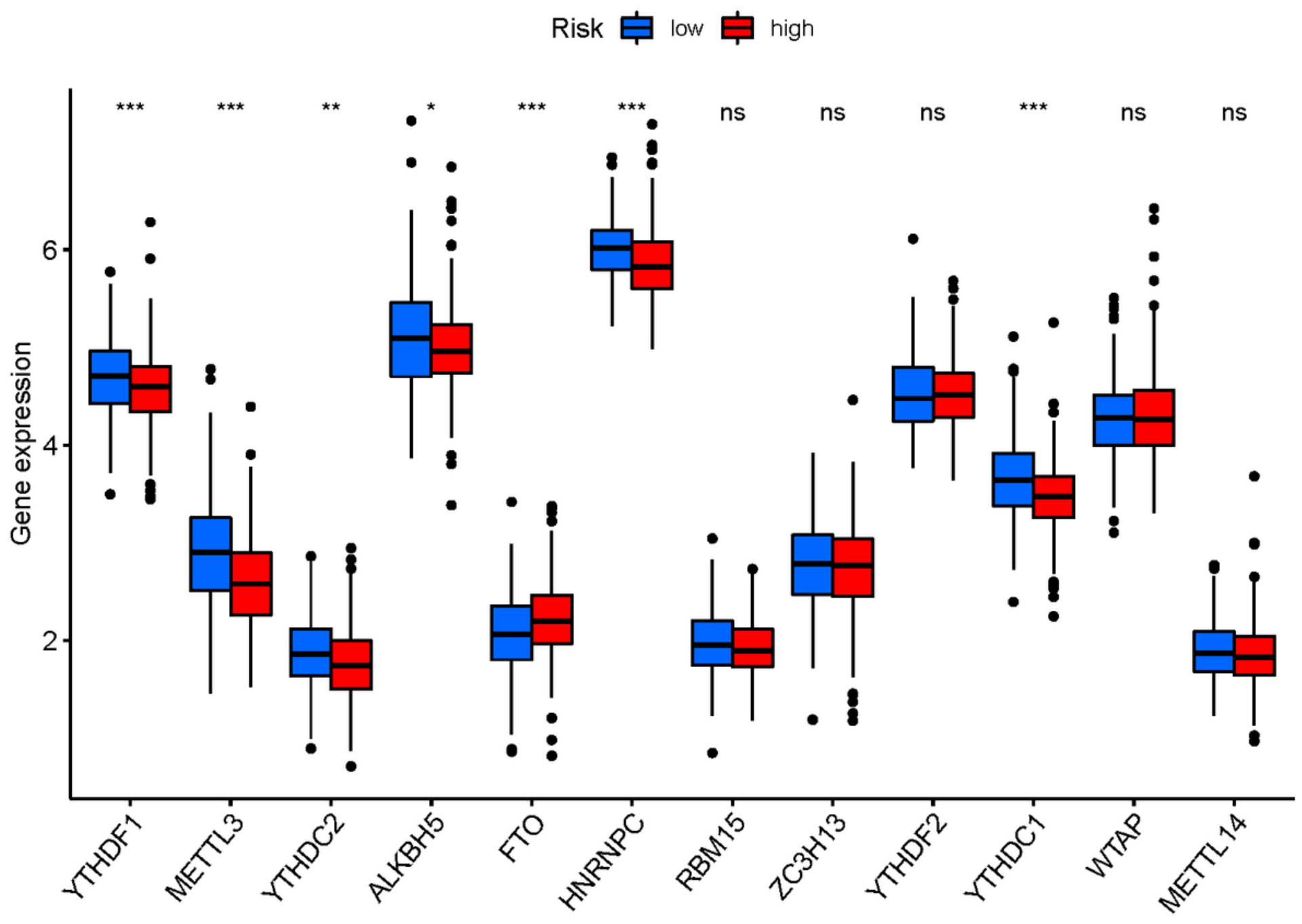

Figure 11

The expression of ferroptosis-related genes in LUSC risk groups with high and low LUSC risk.

\section{Supplementary Files}

This is a list of supplementary files associated with this preprint. Click to download.

- appendix.doc 